\title{
Digital Study of the Thermo-Mechanical Behavior of a Dry Granular Media in a Cell of Annular Shear
}

\author{
Soumia TEYAR, Youcef GUETTECHE \\ University of 20 August 55 Skikda, Mechanical department of Engineering, BP 26, Skikda 21000, Algeria, \\ E-mail: teyar.soumia@yahoo.fr, guetteche@gmail.com
}

crossref $\mathrm{http}: / / \mathrm{dx}$.doi.org/10.5755/j01.mech.24.6.22461

\section{Nomenclature}

$a$ - contact radius; $C$ - specific heat; $F_{\text {ext }}$ - external forces; $g$ - distance between particles; $h$ - time step; $h_{t}$ - height of the slice; $\boldsymbol{H}$ - linear mapping from contact to body level; $H$ - contact conductance; $k_{t h}$ - contact thermal conductivity; $L$ - length of the cylinder; $\boldsymbol{M}$ - mass matrix; $\boldsymbol{M}^{-\mathbf{1}}-$ inverse matrix; $N_{c}-$ contact number; $P$ - percussion; $q-$ configuration vector; $\dot{q}$ - first-time derivative of the configuration vector; $\ddot{q}$-acceleration vector or the second derivative; $Q_{i}^{\text {cond }}$ - transfer of the local heat by conduction; $Q_{i}^{\text {diss }}-$ local generation of the heat.

\section{Introduction}

The study of granular media is of particular interest for the researches in a many scientific fields: physics, mechanics, geophysics, chemistry...The flow of the solid particles which constitute this medium under different operational conditions, carry to a multitude of different behaviors. In particular, the study of the behavior of these particles during shearing carried a more local look at the phenomena of friction, wear and heat generation in the different mechanical systems such as: braking, gearing, machining.

In light of the resolution of the local problem of frictional contact due to dry annular shear where particle shear is indefinite and the rate is high, recent studies have shown that operating conditions play a crucial role in the dynamic properties of granular flows under this type of shear. As an example, F. Chevoir and al. [1, 2] studied the behavior of the granular material in the first layers in the vicinity of the inner cylinder where the localization of the shear. The understanding of this behavior results from the measurement of compactness profiles, speed and constraints, as well as fine size such as the coordination number or the fluctuations of the grains motions. In agreement with the experimental observations, they showed that the evolution of the effective friction of the material to the wall depends on the roughness of the inner cylinder. However, G. Koval [3] found that the strong deformations are observed in this zone of shear localization with a heterogeneity of the distribution of the stress fields. On the other hand, J. Shunying and al [4] realized a direct comparison between the experimental and numerical results by the method of the discrete elements of the sheared materials at different speeds of rotation and pressures. They found that not all the relative particle-level information, such as rigidity, grain size distribution, and damping, is equally important for simulating the shear response.
However, the characteristics of the friction is very important to match the measured forces in the physical system.

J. J. McCarthy and A. Alenzi [5,6] studied the influence of the geometry of the system and the shape of the particles on the quality and quantity of sheared granular flux. As for the different rotational speed and roughness values of the inner cylinder, they found that both of these characteristics have a significant influence on the solid fraction profiles as well as the velocity and granular temperature distributions. C.C. Liao on his part studied the effect of gradient of speed on the experimentally measured granular temperature [7]. The results show that the granular temperatures in a system with both walls rotating in opposite directions, are higher than in a system with walls rotating in the same direction.

The majority of these studies have tried to find the mechanical responses of the contact, without taking into account the thermal aspect. However, there has been recent research done on the analysis of thermal phenomena which occur during the dry shear of particles but in other nonannular cases. We find, for example, the study by M. Filali and al [8] on the influence of the mechanical stresses applied on the granular medium, where they found that the uni-axial compression of the medium serves to reduce its initial volume, therefore its porosity is an increase in the apparent thermal conductivity. Others [9] have studied the influence of the physical properties of the medium on the power dissipated by friction of the particles, as well as the study of the phenomena of friction and diffusion of heat [10]. The analysis of the results obtained show that the profiles of heat and power depend on the nature of the rate of speed accommodation which depends on cohesion.

The evolution of thermal transfers in the sheared granular medium has been studied by V. D. Nguyen and al where they have shown that the roughness of the walls plays a primary role in generating heat by friction [11]. Otherwise the agglomerates (clusters of particles) favor the propagation of heat towards the walls [12]. In addition, T. Champelevier [13] has studied the influence of the parameter of adhesion between the particles on the temperature of the medium. The results of this study show that the temperature of the medium increases with the increase of adhesion and that the heat profiles obtained within the volume of this medium depend on this parameter. Nevertheless, M. Renouf and al [14] studied the influence of particle cohesion. The thermal results obtained show an asymmetry of the temperature profiles for cohesion values used, ie the maximum temperature is located near the zone where the shearing is maximal. The influence of the arrangement of particle on the diffusion of the temperature of the heat source in the medium was studied by J. Riviere 
and al [15] where they showed that the evolution of this temperature depends exclusively on the number of contact that is related to the compactness and therefor how the particles are arranged in the medium.

To complete the mechanical response of the particles during annular shear as well as the difficulties of the experimental realization of the thermal study, we will carry out in this work a 2D numerical study on the thermomechanical behavior of a granular medium in an annular shear cell at shear rate and confining pressure imposed. We will use the Discrete Element Method (MED), and introduce into our numerical model, other parameters that influence in the phenomenon of heat generation by friction such as thermal conductivity, thermal capacity, Young's modulus and pressure. This heat generated in the contact, is transferred by conduction between the particles, according to the model "Thermal Particle Dynamics" proposed by Vargas [16]. Convection is not taken into account in this model because of its weak influence.

This present work is structured as follows: in section 2, we will present the thermo-mechanical formulation used in the model to be realized. Then, we will numerically calculate the different variables of velocity and temperature after the description of the model. A discussion of the results obtained, followed by an analytical analysis, is presented in section 3 . We will finish with a conclusion in the section 4 .

\section{Thermo-mechanical modeling}

\subsection{Numerical formulation}

In the light of the phenomenology study of the granular medium, numerical methods are today more and more developed tools and are precise to characterize physical phenomena at different scales. One of these methods which is widely used, especially in this media, is called discrete elements (Discrete Element Method, DEM) initially developed by Cundall [17]. It is well adapted to simulate the evolution of a particle assembly, taking into account in a discrete way each of them and also their interactions (exerted contact forces). Jean Michel [18] has classified the discrete element methods, according to the function of the laws of interaction applied between particles, into two main categories: the Non Smooth DEM (NSCD) and the Smooth DEM (SD). For this present work, we will use the NSD method which considers shock and friction laws of Coulomb instead of the SD method which considers the continuous and derivable laws.

The mechanical and thermal models used are formulated by the integration scheme of type $\theta$ method and the implicit management of all the contacts.

In order to be able treat simultaneously deal with contact and shock during the same time step, Moreau [19] proposes a rewrite of the equation of dynamics in the sense of distributions. Thus, in the case of a system consisting of rigid particles, this equation is expressed as:

$$
\boldsymbol{M} \ddot{q}(t)=F_{e x t}(t, q, \dot{q})+R(t),
$$

where $\boldsymbol{M}$ is the mass matrix of the granular system $\left(\in R^{n x n}\right), \ddot{q}(t)$ is the acceleration vector or the second derivative of the configuration vector $q, F_{\text {ext }}$ the applied external forces on the particles in contact and $R(t)$ the reactions of the rubbing contact.

The equation of the system is detailed in Jean's initial works [19].

In terms of local variables and with the laws of interaction of the couple $\left(r_{i+1}, u_{i+1}\right)$, the system is rewritten as follows:

$$
\left\{\begin{array}{l}
\boldsymbol{W h} r_{i+1}+u_{i(\text { free })}=u_{i+1} \\
\text { Interaction }\left(r_{i+1}, u_{i+1}\right)
\end{array} .\right.
$$

Where: $\boldsymbol{W}=\boldsymbol{H}^{*} \boldsymbol{M}^{-1} \boldsymbol{H}$ is the operator of Delassus $[19,20]$.

The resolution of the multi-contact problem is a nonlinear Gauss-Seidel type (NLGS) [19].

Eq. (2) is rewritten for a contact $\alpha$ which have a contact number $N_{c}$ as follows:

$$
\sum_{\beta=1}^{N_{C}} W_{\alpha \beta} h r_{i+1}^{\beta}+u_{i(\text { free }, \alpha)}=u_{i+1(\alpha)} .
$$

The law of interaction used between the media particles is the unilateral law with friction without cohesion called IQS_CLB (Inelastic Quasi Shock Law coupled with the Coulomb Friction Law) [20]. For this law, we have taken into account the non-penetration between the particles where the value of the interstice must remain positive $(g \geq 0)$ and the normal component of the local impulse also remains positive by translating with $r_{n} \geq 0$.

Impenetrability condition (Signorini condition) is written as follows:

$$
g \geq 0, r_{n} \geq 0, g \cdot r_{n}=0 .
$$

The thermal model serves to simulate the phenomena of the heat generation by friction and the thermal transfer by conduction. The thermal transfer by radiation and by convection are negligible.

The thermal resolution is represented by the equation of the temperature variation for a single particle during a time step $d t$ :

$$
d T_{i}(t+d t) / d t=\left(Q_{i}^{\text {cond }}+Q_{i}^{\text {diss }}\right) / \rho_{i} C_{i} V_{i} .
$$

With $\rho C_{i} V_{i}$ represents the thermal capacity of the considered particle.

The local generation of the heat $Q_{i}^{\text {diss }}$ between the particles $i$ and its neighbor is calculated by the formula:

$$
Q_{i}^{\text {diss }}=P(\dot{q}(t+d t)+\dot{q}(t)) /(2 d t)
$$

The transfer of the local heat by conduction $Q_{i j}^{\text {cond }}$ between the particles $i$ and its neighbor $j$ is written by:

$$
Q_{i j}{ }^{\text {cond }}=H .\left(T_{i}-T_{j}\right), T_{j}<T_{i} \text {. }
$$

Where $\left(T_{i}-T_{j}\right)$ is the temperature difference between the contact media of the spheres " $i$ " and " $j "$. and $H_{i j}$ is the conductance of the contact between the two spheres " $i$ " and " $j "$, based on the Yovanovich works [21] its value is 
calculated in the two-dimensional model according to the elastic law of Hertz by the formula:

$$
H_{2 D}=2 k_{t h} \sqrt{2 a L}=2 k_{t h}\left(8 r_{n} R^{\prime} / \pi L E^{\prime}\right)^{1 / 4} .
$$

Where: $R^{\prime}, r_{n}$ and $E^{\prime}$ are respectively the radius effective, the normal reaction in contact and the module d'Young effective.

\subsection{Numerical simulation}

\subsubsection{Description of the model}

The geometry of the model realized in this numerical study "Couette" type in 2D (Fig.1) has two cylinders: a movable inner cylinder, which rotates with a rotation speed $\omega$, discretized by disks of diameter of 0.6 $\mathrm{mm}$, which simulate balls; a fixed external cylinder (pneumatic membrane) on which is applied a normal peripheral pressure $P 1$, in order to impose the balls' shear (discs, particles) of the granular media.

The granular media has 7536 (rigid particles calculated according to the geometry) in the form of discs, with an average radius $R_{\text {moy }}=0.25 \mathrm{~mm}$.

To simulate particles shear, the geometry of the model is subjected to impose solicitations: a peripheral pressure $P 1$ applied to the fixed outer cylinder and a rotation of the moving inner cylinder with a velocity $\omega$ (Fig.1).

The annular geometry is defined by the radius of the inner $R_{i n}$ and outer $R_{\text {ext }}$ cylinders distributed uniformly with a dispersion of $10 \%$. The coefficient of friction between particle- particle $\mu_{p-p}$ and particle-cylinder $\mu_{p-c}$ was chosen value of 0,5 . The simulation time is divided into increments of $\Delta t$ (step) equal to $10^{-5} \mathrm{~s}$ ensuring a good compromised between CPU time and solution accuracy.

The numerical characteristics used for the simulations are presented in the Table 1.

\section{Recapitulatory of the numerical characteristics of the model}

\begin{tabular}{|l|l|}
\hline Minimum radius of the balls $R_{\min }, \mathrm{mm}$ & 0.2 \\
\hline Maximum radius of the balls $R_{\max }, \mathrm{mm}$ & 0.3 \\
\hline Outside radius of the Couette $R_{\text {ext }}, \mathrm{mm}$ & 50 \\
\hline Interior radius of the Couette $R_{\text {int }}, \mathrm{mm}$ & 10 \\
\hline Step of time $\Delta t, \mathrm{~s}$ & $10^{-05}$ \\
\hline Simulation time $t_{\text {sim }}, \mathrm{s}$ & 10 \\
\hline Particle-particle friction coefficient $\mu_{p-p}$ & 0.5 \\
\hline Particle-cylinder friction coefficient $\mu_{p-c}$ & 0.5 \\
\hline
\end{tabular}

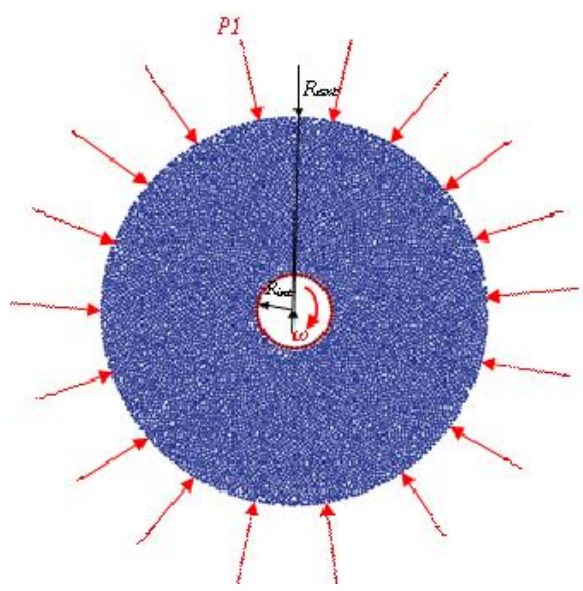

Fig. 1 Schematization of the simulated model

\subsubsection{Calculation of velocities and temperatures}

To measure the different average values (velocity, temperature) per slice through the granular volume (phase discretized). Due to the symmetry, we only need to study a quarter of the geometry that we discretized in slices in the radial direction (Fig. 2).

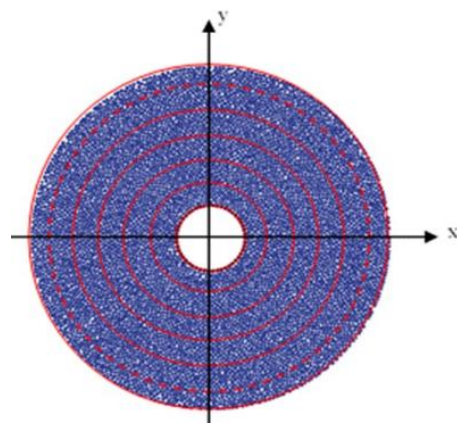

a

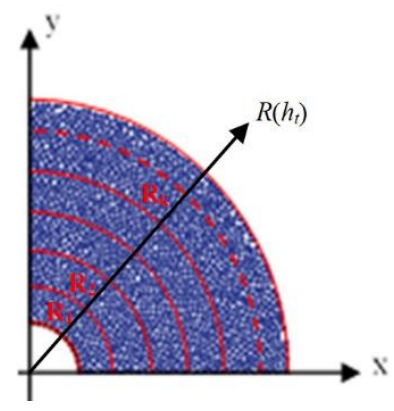

b
Fig. 2 Discretization in the slices of: (a) the geometry and (b) quarter of the geometry

\section{Discussion of results}

\subsection{Influence of particle material}

The imposed conditions for simulating our model are respectively: the peripheral pressure $P 1=10^{+2} \mathrm{~Pa}$ and the rotation speed $\omega=5 \mathrm{rv} / \mathrm{min}$. They are applied to the three selected materials of the granular media particles: steel, copper and aluminum.

The thermo-mechanical characteristics of these materials are shown in the following table:

Table 2

Media particles thermo-mechanical characteristics

\begin{tabular}{|c|c|c|c|c|}
\hline Steel & $\begin{array}{c}\text { Density } \\
\rho\left[\mathrm{kg} / \mathrm{m}^{3}\right]\end{array}$ & $\begin{array}{c}\text { Thermal } \\
\text { conductivity } \\
\lambda\left[\mathrm{w} \cdot \mathrm{m}^{-1} \cdot \mathrm{K}^{-1}\right]\end{array}$ & $\begin{array}{c}\text { Thermal } \\
\text { capacity } \\
C p \\
{[\mathrm{~J} / \mathrm{kg} . \mathrm{K}]}\end{array}$ & $\begin{array}{c}\text { Young's } \\
\text { modulus } \\
E\left[\mathrm{~N} / \mathrm{m}^{2}\right]\end{array}$ \\
\hline Copper & 8900 & 80 & 400 & $213.10^{9}$ \\
\hline Aluminum & 2698 & 238 & 929 & $69.10^{9}$ \\
\hline
\end{tabular}

During the execution of the model's program, the evolution of the temperatures of the granular media is obtained by the visualization of a video, from which we take a screenshot of the simulation desired time.

The colors are assigned according to the spectrum of the light, blue for a temperature of $T=293 \mathrm{~K}$ until red for $T_{\max }$.

Fig. 3 shows an increase in the temperature in the first layers in the vicinity of the inner cylinder. This is due to the fact that the velocity of the particles is maximum at this point, hence the particles shearing is important, which causes a warming by friction.

For the dispersion of hot and cold particles, we remark in this figure red particles (maximum temperatures, $\left.T_{\max }\right)$ and others less hot (intermediate colors of the spectrum of white light). What is normal is the presence of red particles near the inner cylinder where the friction is maximum, therefore of maximum temperature. However, the abnormal presence of red balls dispersed away from the inner cylinder, can be explained by the fact that the 
migration time of a temperature particle $(T)$ is less than the thermal exchange time.

It is difficult to examine the qualitative and quantitative differences for the two cases of aluminum and copper, for this we will show this difference by the temperature profiles obtained hereafter (Fig. 5).

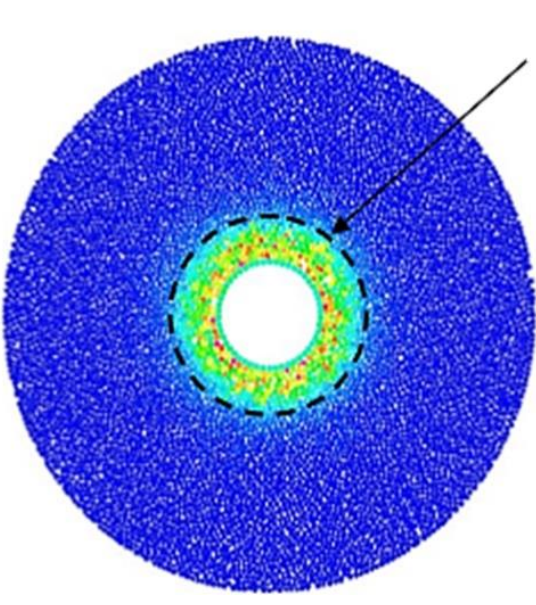

a

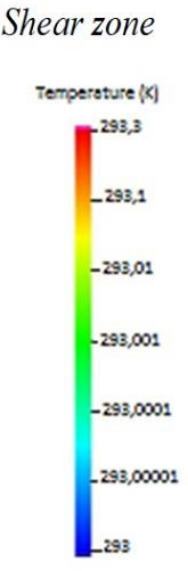

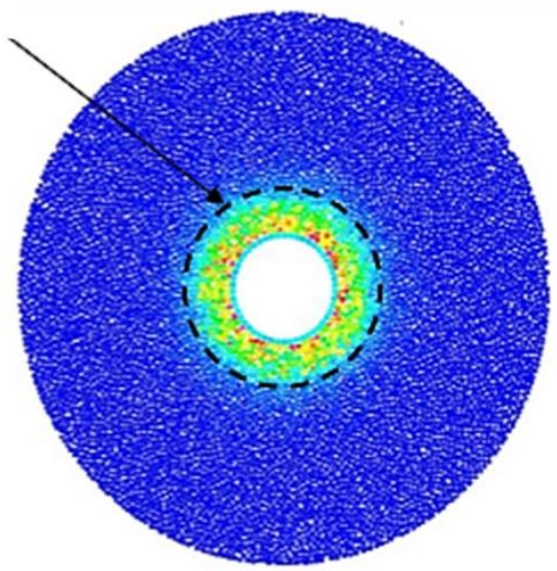

b

Fig. 3 Visualization finally of simulation (after 10 seconds) for $P 1=10^{+4} \mathrm{~Pa}$ and $\omega=5 \mathrm{tr} / \mathrm{min}$ in the case: a) Copper; b)

Aluminum

According to the profiles of the radial velocities obtained for the three selected materials (Fig. 4), we remark two main zones:

Zone 1: is the zone near the inner cylinder that is perturbed, hence the shear is maximum. The velocity profiles decreased generally in the radial direction, but sawtooth.

Zone 2: is the zone away from the inner cylinder which is more stable, where the particles velocity values decrease until zero at the contact with the outer cylinder. The particles flow regime in this zone is practically quasistatic.

The extreme peaks observed in this zone physically show the instability of the flow of particles near the source of the movement.

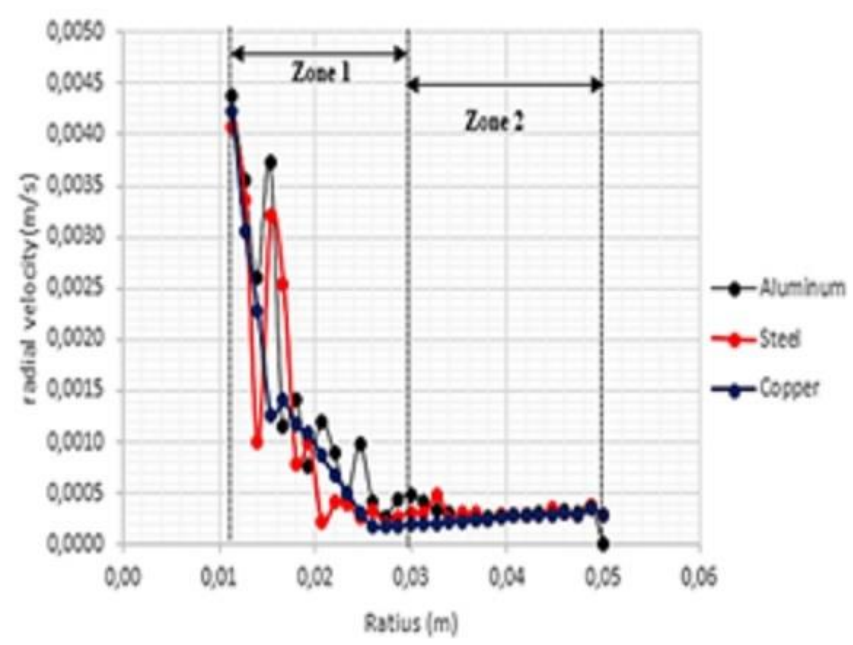

Fig. 4 Profiles of the radial velocity for the three materials (Aluminum, Steel, Copper)

The gaits of the temperature profiles obtained (Fig. 5) for the three materials have the hyperbolic form. We distinguish two main areas:

- in Zone 1 , we remark that the maximum value of the particles temperature is found near the inner cylinder where the radial velocity is maximum (Fig. 5), which is decreasing exponentially;

- in Zone 2, we observe that this zone occupies a large part of the temperature's curve (Fig. 5). It is in constant linear form, which shows that the temperature is stabilized in this zone.

This is due to the diffusion of heat by the particles' contacts, indicating a thermal equilibrium between the particles.

By comparing the temperature patterns of the three materials (Fig. 5), we note that the maximum temperature value for aluminum particles is slightly larger than those of copper and steel because of the ratio $\left(C_{p} / E\right)$.

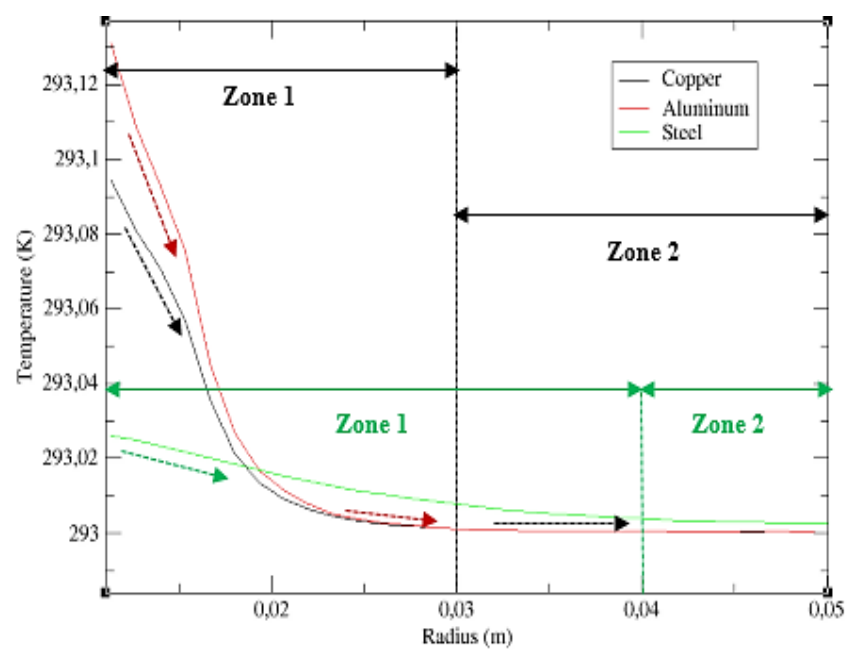

Fig. 5 Profiles of temperature $T(R)$ for the different materials

\subsection{Influence of pressure}

In this part, we present the influence of imposed pressure on the granular media thermo-mechanical behavior.

We have realized simulations for the cases of a less diffusive material (steel) and a more diffusive material 
(copper), with the following conditions:

- rotation velocity of the inner cylinder: $\omega=5 \mathrm{rv} / \mathrm{min}$;

- pressure on the outer cylinder periphery $P 1$ equal to $10^{+2} \mathrm{~Pa}$ and $10^{+4} \mathrm{~Pa}$.

The choice of these values is justified by the respect of the particles interpenetration condition of the particles $\delta$ which must be less than $0.1 \%$ of the average particles radius.

In the case of copper particles, the allure of the obtained temperature profiles (Fig. 6) shows that:

- near the inner cylinder, the obtained maximum temperature (red circle) in the case of the pressure $P 1=$ $10^{+4} \mathrm{~Pa}$ is greater than that of $P 1=10^{+2} \mathrm{~Pa}$;

- the Zone 1 width increases with the increasing of the pressure $P 1$.

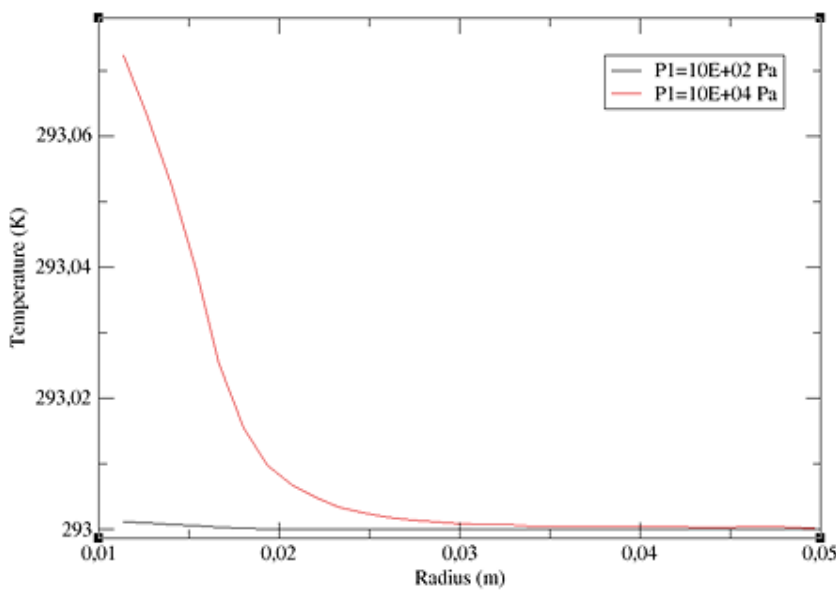

Fig. 6 Temperature profiles of the Copper particles $T(R)$ of the values of the peripheral pressure $P 1: 10^{+2} \mathrm{~Pa}$ and $10^{+4} \mathrm{~Pa}$

According to the allure of the obtained temperature profiles in the case of steel particles (Fig. 7), we remark that: - near the inner cylinder, the obtained maximum temperature (red circle) in the case of the pressure $P 1=$ $=10^{+4} \mathrm{~Pa}$ is greater than that of $P 1=10^{+2} \mathrm{~Pa}$;

- the difference between the maximum temperatures for the two pressure values is less important in the case of steel than in that of copper (Fig.6);

- such as in the case of copper (Fig.6), a low pressure generates and diffuses a low heat;

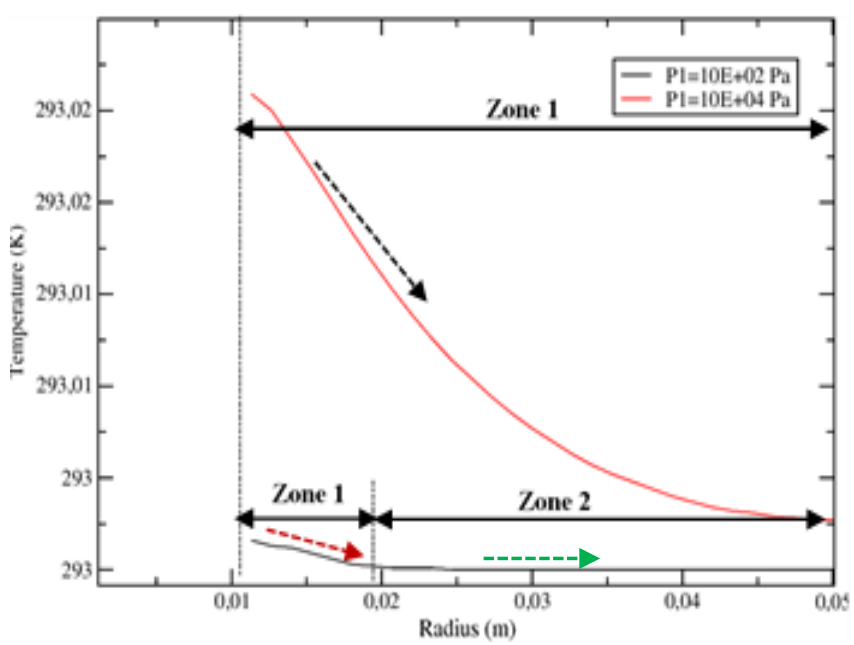

Fig. 7 Temperature profiles $T(R)$ of steel media for the two values of the pressure $P 1: 10^{+2} \mathrm{~Pa}$ and $10^{+4} \mathrm{~Pa}$
- in the case of the high pressure $P 1$, the allure of the temperature profile is decreasing continuously within an exponential form along the radius of the medium $\left(R_{\text {ext }}{ }^{-}\right.$ $R_{\text {int }}$;

- in the case of the low pressure $P 1$, the slope of the temperature curve is very low (red arrow), mostly in Zone 2 .

\subsection{Analytical analysis}

According to the temperature profiles obtained for the two treated cases, we can formulate analytically the influence of the nature of the particle material and the tribological solicitations on the thermal behavior of the particles as follows:

As previously mentioned in the section. 3.3, the temperature values of the discretized medium decreases exponentially along the radius $\left(R_{\text {ext }}-R_{\text {int }}\right)$ (Fig. 5, 6 and 7), we can then analytically write the equation of temperature in the form:

$$
T\left(R_{k}\right)=T_{0}+A_{k} e^{-R_{k}}=T_{0}+\Delta T_{k} e^{-R_{k}} .
$$

With: $R_{\text {int }} \leq R_{k} \leq R_{\text {ext }}$ and $k=1, m(m:$ number of the slice); $\Delta T_{k} \cdot e^{-\mathrm{R}_{\mathrm{k}}}$ : the variation of the temperature along the radius $« R_{k} » ; R_{k}$ :is radius of the slice $« k »$.

Knowing that the initial thermal conditions imposed are: at $t=0 \mathrm{~s}, T_{0}=T\left(R_{0}\right)=T\left(R_{\text {ext }}\right)=293 \mathrm{~K}$.

As our geometry is divided into several slices of the same thickness $\left(h_{s}\right)$ (Fig. 2), the overall heat flow along the radius $« R_{k} »$ can be written by the equation:

$$
Q_{\text {glob }}=\sum_{k=1}^{m} Q_{k}
$$

With: $Q_{k}$ is heat flow generated by friction for each slice $k$ $k$.

$$
Q_{k}=\mu_{k} \cdot F_{N k} \cdot V_{g k}=F_{T k} \cdot V_{g k} .
$$

As previously mentioned (Eqs. 7 and 11), the generated heat flow is written:

$$
Q_{k \text { cond }}=H \cdot \Delta T_{k}=\mu_{k} \cdot F_{N k} \cdot V_{g k}=F_{T k} \cdot V_{g k} .
$$

so:

$$
\Delta T_{k i}=\left[\frac{F_{T k} \cdot V_{g k}}{H}\right]=\left[\frac{T\left(R_{k i}\right)-T_{0}}{e^{-R_{k}}}\right] .
$$

We can therefore rewrite the equation of temperature as follows:

$$
T\left(R_{k}\right)=T_{0}+. \Delta T_{k} e^{-R k}=T_{0}+\left[\frac{F_{T k} \cdot V_{g k}}{H}\right] \cdot e^{-R_{k}} .
$$

With: $F_{T k}$ is average tangential force of the slice particles « $k » ; Q_{k c o n d}$ is the flow of heat transferred by conduction for each slice $« k » ; T\left(R_{k}\right)$ is the radial temperature for each slice $« k » ; \mu_{k}$ is average friction coefficient of the 
slice particles « $k » ; F_{N k}$ is average normal force of the particles of the slice « $k » ; V_{g k}$ is average sliding velocity of the slice particles $« k »$.

According to Eq. (13), we remark that:

the evolution of the temperature follows the pressure (the normal force) applied, in the case where the value of " $H$ " is constant (same type of material);

- the maximum temperature for a more conductive material (copper) is greater than for a less conductive material (steel). This shows a great influence of the thermal conductivity on the temperature evolution of the chosen material.

\section{Conclusion}

After this parametric study of the "Couette" model, applied to our numerical model, we can conclude the following points:

- the allure profiles obtained are identical to those obtained in François Chevoir and Alain Corfdir [1] and David Richard works [10];

- the increase in the pressure $P 1$ causes an increase in the friction between the particles so an increase in heat generation which breeds an increase in the maximum temperature of the medium $T_{\max }$;

- the softer the material, the more sensitive it is to strong stresses, the higher the temperature;

- the higher the thermal capacity via the Young's modulus $E$ is going to be weak, the higher the maximum temperature of the medium is going to be higher;

- the heat generation is mainly due to pressure and friction between particles in the shear zone (Zone 1).

\section{Acknowledgement}

This study was carried out within the Contact and Structural Mechanics Laboratory (LaMCoS) of INSA Lyon as part of long-term training. A gratefully thanks to $\mathrm{Mr}$ Professor Yves Berthier, and $\mathrm{Mr}$ Mathieu Renouf respectively CNRS Research Director and CNRS researcher for their help that allowed me to use the numerical tool for this work

\section{References}

1. GDR MIDI. 2004. On dense granular flows, The European Physical Journal E14: 341-365. https://doi.org/10.1140/epje/i2003-10153-0.

2. Canou, J.; Chevoir, F.; Corfdir, A.; Duhamel, D.; Dupla, J. C.; Roux, J. N. 2007. UMR NAVIER, Comportement mécanique des matériaux granulaires : apports des simulations numériques discrètes. https://trid.trb.org/view/937388.

3. Koval, G.; Roux, J.; Cofdir, A.; Chevoir, F. 2009. Annular shear of cohesionless granular materials: from inertial to quasistatic regime, Physical Review E 79:1-18. https://doi.org/10.1103/PhysRevE.79.021306.

4. Ji, S.; Hanes, D. M.; Hayley, H. 2009. Comparisons of physical experiment and discrete element simulations of sheared granular materials in an annular shear cell, Shen State Key Laboratory of Structural Analysis for Industrial Equipment, Dalian University of Technology, USA Civil and Environmental Engineering; Clarkson University, Potsdam, USA.

https://doi.org/10.1016/j. mechmat. 2009.01.029.
5. McCarthy, J. J.; Jasti, V. b.; Marinack, M.; Higgs, C.F. 2010. Quantitative validation of the discrete element method using an annular shear cell, Powder Technology $203: 70-77$. https://doi.org/10.1016/j. powtec. 2010.04.011.

6. Alenzi, A.; Marinack, M.; Higgs, C.F.; McCarthy, J.J. DEM validation using an annular shear cell.2013. Powder Technology 248:131-142. https://doi.org/10.1016/ j. powtec. 2013.05.003.

7. Liao, C.-C.; Hsiau, S.-S.; Yu, W.-J. 2012. The influence of driving conditions on flow behavior in sheared granular flows, International Journal of Multiphase Flow 46: 22-31.

https://doi.org/10.1016/j.ijmultiphaseflow.2012.06.003.

8. Filali, M. 2006. Conductivité thermique apparente des milieux granulaires soumis à des contraintes mécaniques: modélisation et mesure. $\mathrm{PhD}$ thesis, Institut $\mathrm{Na}-$ tional Polytechnique de Toulouse.

http://ethesis.inp-toulouse.fr/archive/00000294.

9. Richard D.; Iordanoff I.; Berthier Y.; Renouf M.; Fillot N. 2007. Friction coefficient as a macroscopic view of local dissipation, 129: 829-835. https://doi.org/10.1115/1.2768083.

10. Richard, D. 2008. Thermique des contacts avec troisième corps solide-Modélisation et compréhension des phénomènes de frottement et de diffusion de chaleur par la méthode des éléments discrets, Thèse de Doctorat de l'INSA de Lyon.

http://www.sudoc.fr/131859919.

11. Nguyen, V. D.; Cogné, C.; Guessasma, M.; Bellenger, E.; Fortin, J. 2009. Discrete modeling of granular flow with thermal transfer: application to the discharge of silos. Applied Thermal Engineering 29:1846-1853. https://dx.doi.org/10.1016/j.applthermaleng.2008.09.009.

12. Nguyen, V. D.; Brunel, J-F.; Magnier, V.; Dufrénoy, P.; Coorevits, P. 2013. Rôle des rugosités et des plaques portantes dans un modèle discret d'interface de contact d'un système de freinage, CSMA 2013, 11e Colloque National en Calcul des Structures. https://hal.archives-ouvertes.fr/hal-01722058.

13. Champelovier, T.; Renouf, M. 2016. Modèle multiphysiques par éléments discrets. https://hal.archives-ouvertes.fr/hal-01413972.

14. Renouf, M.; Cao, H. -P.; Nhu, V. -H. 2011. Multiphysical modeling of third-body rheology, Tribology International 44: 417-425. https://doi.org/10.1016/j.triboint.2010.11.017.

15. Riviere, J.; Renouf, M.; Berthier, Y. 2013. Simulation du comportement thermique d'un milieu continu équivalent par méthode des éléments discrets, CSMA 2013, 11e Colloque National en Calcul des Structures. https://hal.archives-ouvertes.fr/hal-01717076.

16. Vargas, W. L.; McCarthy, J. J. Heat conduction in granular materials, 2001. Aiche journal 47: 1052-1059 https://doi.org/10.1002/aic.690470511.

17. Cundall, P. A.; Strack, O.D.L. 1979. A discrete numerical model for granular assemblies, Geotechnique, 29(1): 47-65. https://doi.org/10.1680/geot.1979.29.1.47.

18.Jean, M. 1999. The non-smooth contact dynamics method, Compt. Methods Appl. Math. Engrg, 177: 235257.

https://doi.org/10.1016/S0045-7825(98)00383-1. 
19. Moreau J. J. 1988. Unilateral contact and dry friction in finite freedom dynamics, In J.J. Moreau and eds. P.D. Panagiotopoulos, editors, Non Smooth Mechanics and Applications, CISM Courses and Lectures, 302: 182. https://dx.doi.org/10.1007/978-3-7091-2624-0.

20. Tholen, A. R. 1992. Particle-particle interaction in granular material. In: I. L. Singe, H.M. Pollock, Fundamentals in friction: macroscopic and microscopic processes. Dordrecht, Kluwer Academic Publishers, 25: 95-110. https://link.springer.com/chapter/10.1007/978-94-0112811-7_6.

21. Yovanovich M. M. 1967. Thermal contact resistance across elastically deformed spheres, Journal of Spacecraft and Rockets 4(1): 119-122. https://doi.org/10.2514/3.28821.

\section{S. Teyar, Y.Guetteche}

DIGITAL STUDY OF THE THERMO-MECHANICAL BEHAVIOR OF A DRY GRANULAR MEDIA IN A CELL OF ANNULAR SHEAR

\section{S u m m a r y}

In the aim to understand the thermo-mechanical phenomenology of the dry granular media due to the grains' flow nature of (rheology) or particles that constitute this medium. We will illustrate in this present article a numerical study by the discrete elements (MED) method on the thermo-mechanical behavior of a set of rigid particles sheared in an annular cell. This study is focused on the modeling of local friction between particles, from the generation of heat and its diffusion by conduction. We realized out a two-dimensional (2D) numerical model of type Couette geometry to simulate the different thermo-mechanical quantities such as: flow velocity and media temperature. This modeling is implemented within the platform used LMGC90, which is based on the NSCD (Non-Smooth Contact Dynamics Method) approach.

In the First time, we'll study the various realistic physical quantities of the contact's rheology (type of particles material, the applied pressure and the shear velocity). Then we will continue by the analytical analysis and interpretation of the obtained results.

Keywords: friction, annular shear, dry granular media, generation of heat, conduction heat transfer, NSCD, thermomechanical behavior.

Received August 25, 2018

Accepted December 12, 2018 